\title{
Does social support modify the relationship between food insecurity and poor mental health? Evidence from thirty-nine sub-Saharan African countries
}

\author{
Muzi Na ${ }^{1, *}$, Meghan Miller ${ }^{2}$, Terri Ballard ${ }^{3}$, Diane C Mitchell ${ }^{1}$, Yuen Wai Hung ${ }^{4}$ and \\ Hugo Melgar-Quiñonez ${ }^{2}$ \\ 'Department of Nutritional Sciences, College of Health and Human Development, The Pennsylvania State University, \\ 108C Chandlee Lab, University Park, PA 16801, USA: ${ }^{2}$ McGill Institute for Global Food Security, School of Human \\ Nutrition, McGill University, Montreal, Quebec, Canada: ${ }^{3}$ Independent Consultant, Montebuono, Rieti, Italy: ${ }^{4}$ The \\ Methodology Center, College of Health and Human Development, The Pennsylvania State University, University Park, \\ PA, USA
}

Submitted 12 July 2018: Final revision received 27 August 2018: Accepted 14 September 2018: First published online 5 November 2018

\begin{abstract}
Objective: The present study aimed to determine the relationship among food insecurity, social support and mental well-being in sub-Saharan Africa, a region presenting the highest prevalence of severe food insecurity and a critical scarcity of mental health care.

Design: Food insecurity was measured using the Food Insecurity Experience Scale (FIES). Social support was assessed using dichotomous indicators of perceived, foreign perceived, received, given, integrative and emotional support. The Negative and Positive Experience Indices (NEI and PEI) were used as indicators of mental well-being. Multilevel mixed-effect linear models were applied to examine the associations between mental well-being and food security status, social support and their interaction, respectively, accounting for random effects at country level and covariates.

Participants: Nationally representative adults surveyed through Gallup World Poll between 2014 and 2016 in thirty-nine sub-Saharan African countries ( $n$ 102 235). Results: The prevalence of severe food insecurity was $39 \%$. The prevalence of social support ranged from 30 to $72 \%$ by type. In the pooled analysis using the adjusted model, food insecurity was dose-responsively associated with increased NEI and decreased PEI. Perceived, integrative and emotional support were associated with lower NEI and higher PEI. The differences in NEI and PEI between people with and without social support were the greatest among the most severely food insecure.

Conclusions: Both food insecurity and lack of social support constitute sources of vulnerability to poor mental well-being. Social support appears to modify the relationship between food security and mental well-being among those most affected by food insecurity in sub-Saharan Africa.
\end{abstract}

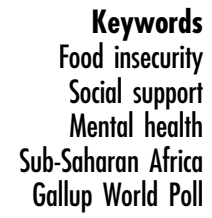

The WHO describes mental health as more than the absence of pathology, but as 'a state of well-being in which every individual realizes his or her own potential, can cope with the normal stresses of life, can work productively and fruitfully, and is able to make a contribution to her or his community ${ }^{\text {(1) }}$. Common mental health disorders are responsible for significant losses in health worldwide, with depression ranked first among contributors to global disability and anxiety ranked sixth ${ }^{(2)}$. The number of people living with the most common mental health problems has increased over the past decade, reflecting both the growth and ageing of the world population, as the prevalence of depression peaks in older adulthood $^{(2)}$

Poor mental health is associated with diverse social, psychological and biological risk factors ${ }^{(1,3)}$. Among socioeconomic risk factors, food insecurity, or lack of access to adequate food, is considered an important social determinant of health ${ }^{(4)}$ and has been linked to a wide range of adverse mental and physical health conditions ${ }^{(5,6)}$. In a systematic review examining the relationship between socio-economic disparities and common mental disorders in low- and middle-income countries, food insecurity was shown to be a strong risk factor ${ }^{(7)}$. An analysis of data from 
149 countries found consistent associations between food insecurity and poor mental health after controlling for potential socio-economic confounders ${ }^{(8)}$. Food insecurity may contribute to different types of stress, which in turn may lead to poor mental health through three possible pathways: (i) physical stress, due in part to poor nutrition and micronutrient insufficiency; (ii) psychological stress, due to anxiety about obtaining enough food; and (iii) social stress, including shame, stigma and isolation, awareness of the social unacceptability of food behaviours and interpersonal tension in the household ${ }^{(9)}$.

Another key social determinant of health, social support has been studied across disciplines for its effect on mental and physical well-being. A distinction is commonly made between support that is perceived to be available and support that is enacted or actually received. Social support can also be categorized as intentionally provided in response to a specific need or as an underlying supportive condition (intentionally or unintentionally) provided by belonging to a social group. Furthermore, supportive functions are often categorized as 'emotional' (reassurance, empathy, listening), 'instrumental' ('practical' things like money, goods, tools, services), 'informational' (advice, guidance), 'companionship' (a sense of belonging) and 'validation' (feedback about one's status in relation to others) ${ }^{(10)}$. These distinct types of support may act in very different ways to affect health outcomes and may be more or less influential, depending on an individual's situation and whether the health condition of interest is acute or chronic $^{(11)}$. Two frequently cited models propose how different support types and functions may work to affect mental health: (i) the main effects model hypothesizes that social support directly improves health through promoting healthy behaviours and altering neuroendocrine responses to influence emotional states, regardless of the presence or severity of specific stress factors; and (ii) the stress-buffering model proposes that social support offsets the negative impact of stressors by altering the cognitive, emotional or behavioural response or by affecting how people appraise their situation ${ }^{(10)}$.

Although evidence demonstrating the separate influences of food insecurity and social support on health has accumulated, studies have defined and measured the two factors in different ways and few have explored how they may interact to affect well-being. The limited number of studies that explored both have focused narrowly on specific health outcomes, including obesity ${ }^{(12)}$ and sickle cell disease $^{(13)}$, while those that have considered mental health outcomes focused primarily on depressive symptoms among sub-populations ${ }^{(14-16)}$.

The prevalence of food insecurity in sub-Saharan Africa is the highest in the world and continues to increase, with $31 \%$ of the population experiencing severe constraints in accessing adequate food, according to the most recent estimates in 2016 ${ }^{(17)}$. In many sub-Saharan African countries, complex family structures are common ${ }^{(18)}$. Many live in closely knit communities and rely on their social networks, such as friends, relatives and neighbours, for both tangible and intangible support ${ }^{(19)}$. Formal public social protection systems are often weak in this region, leading people to rely more heavily on informal (non-governmental) social support structures to cope in the face of shocks ${ }^{(19)}$. Individuals suffering from mental illness in Africa face a particularly virulent combination of socioeconomic and cultural conditions: government spending on mental health care is very low, with the majority of countries in Africa devoting less than $1 \%$ of their total health-care budget to treating mental conditions. Shortages of trained mental health professionals as well as unavailability of medications such as antidepressants present a supply-side obstacle to treatment, while financial constraints and social stigma may discourage patients from seeking the care they need ${ }^{(3)}$. The African region has a higher prevalence of depressive disorders (5.4\%) than the global average ${ }^{(2)}$; however, this number is likely to underestimate the true burden of poor mental health because it is limited to one category of mental disorder and does not consider low rates of diagnosis.

Given that sub-Saharan Africa faces both food insecurity and mental health crises, the present study focuses on this most vulnerable region and aims to explore the association between food insecurity, social support and mental well-being, using nationally representative survey data from thirty-nine countries collected across three years (2014-2016). Building on the global analysis by Jones ${ }^{(8)}$, which used data from the same survey and employed the same measures of both food insecurity and mental wellbeing, the present study contributes to the ongoing effort to recognize and understand the non-nutritional psychological consequences of food insecurity. The distinction made among different types of social support is an important addition, as it allows for the consideration of multiple pathways by which social support may act to modify the association between food security status and mental well-being.

\section{Methods}

\section{Study area and population}

The present study analysed data from the 2014-2016 Gallup World Poll (GWP) in thirty-nine sub-Saharan African countries. Countries not surveyed in GWP 2014-2016 were Cabo Verde, Comoros, Equatorial Guinea, Eritrea, Gambia, Guinea-Bissau, São Tomé and Príncipe, Seychelles and Swaziland. The GWP is an annual survey of adult (aged $\geq 15$ years) individuals' perceptions, opinions and experiences, conducted in over 140 countries since 2006. The detailed methodology of GWP sampling and variable coding is available elsewhere ${ }^{(20)}$. In brief, nationally representative samples of $\sim 1000$ households were selected using a stratified multistage cluster sampling 
design. In the first stage, 100-126 clusters of households in urban and rural areas were selected based on probability proportional to population size and these clusters were considered as primary sampling units. In the second stage, an average of eight households were selected within each primary sampling unit by a random route procedure and the respondents within the selected households were randomly chosen using a Kish Grid method.

Face-to-face interviews were carried out in all thirtynine countries in all years. In some countries and in specific survey years, there was approximately 3-44\% of the total population excluded from the sampling process due to security concerns ${ }^{(21)}$. The sample size by year for each of the thirty-nine countries and the proportion of the national population excluded are listed in the online supplementary material, Supplemental Table 1.

\section{Mental well-being variables}

Two aggregated indices created by Gallup are used to measure general mental well-being ${ }^{(20)}$ (see online supplementary material, Supplemental Table 2). The Negative Experience Index (NEI) is a composite index of five questions regarding the experience of physical pain, worry, sadness, stress and anger during the previous day. The Positive Experience Index (PEI) is a similar composite index that includes questions about feeling well-rested, being treated with respect, smiling, laughing, learning or doing something interesting, and experiencing enjoyment during the preceding day. Each question is initially scored dichotomously as ' 1 ' if experienced or ' 0 ' if not experienced. The mean score of the five questions under the domain of either NEI or PEI is multiplied by 100 to produce the composite indices (both range from 0 to 100) ${ }^{(21)}$. The indices are calculated at the individual level, with lower NEI and higher PEI scores indicating better mental well-being. GWP reports that both NEI and PEI have satisfactory internal consistency (Cronbach's $\alpha=0.80$ for NEI and 0.91 for PEI) and expected correlation with other socio-economic measures ${ }^{(20)}$. In the current analysis, respondents who answered 'don't know' or 'refused to answer' to any of the five questions that compose NEI or PEI, respectively, were excluded from the outcomespecific analysis.

\section{Adult food insecurity variables}

Since 2014, the GWP has included the eight-item Food Insecurity Experience Scale (FIES) survey module to measure individual-level food insecurity (see online supplementary material, Supplemental Table 2). The FIES is a validated experience-based metric of access to food that captures three domains of behaviours and experiences related to increasing difficulties in food acquisition over a reference period of 12 months: (i) uncertainty or anxiety over obtaining food (worrying about not have enough food to eat); (ii) changes in quality of the diet (not being able to eat healthy and nutritious food, eating only a few kinds of foods); and (iii) reductions in quantity of food consumed (skipping a meal, eating less than the respondent thought he/she should, his/her household running out of food, being hungry but not eating, and going without eating for a whole day) ${ }^{(22)}$. In the current analysis, an answer of 'yes' to a given question was recorded as 1 and 'no' as 0 . A respondent's raw score was calculated by summing the eight dichotomous items, producing a variable that ranged from 0 (no food insecurity experiences) to 8 (affirmed all eight experiences). A raw score was considered 'complete' if respondents answered 'yes' or 'no' to all eight questions, while those with any 'don't know' or 'refused' responses were excluded from the analytic sample. Individuals were further classified into four groups according to commonly used ranges of raw scores as follows: $0,1-3,4-6$ and 7-8. These categories represent four levels of food insecurity severity, with 7-8 being the most severe ${ }^{(22)}$.

\section{Social support variables}

Six questions from the GWP were considered to capture aspects of the broad concept of social support (SS; see online supplementary material, Supplemental Table 2). The distinct types of support represented by the questions were: (i) perceived SS; (ii) foreign perceived SS; (iii) received SS; (iv) given SS; (v) integrative SS; and (vi) emotional SS. The last question on emotional support was dichotomized to a value of ' 0 ' if the answer was 'strongly disagree', 'disagree' or 'neutral', or to a value of ' 1 ' if the answer was 'agree' or 'strongly agree'. An exploratory analysis of the relationship between the SS variables indicated that the six items may have measured different dimensions of social support. Therefore, the decision was made to analyse them individually and not combined into a global SS variable because (i) the Cronbach's $\alpha(0.43)$ and correlation (ranges 0.01-0.44) were low among the six SS items and (ii) the principal component analysis and parallel analysis did not show any significant clustering factors (Supplemental Fig. 1).

\section{Statistical analysis}

The statistical software package STATA/SE $15 \cdot 0$ was used to analyse the data. Proportions and means describing the respondents' demographic characteristics, FIES raw score, food insecurity severity category and SS variables were calculated after adjusting for the complex sampling design using the 'svy' command. Standard errors and 95\% confidence intervals were estimated using the Taylor series linearization methods ${ }^{(23)}$

To investigate the relationship between food insecurity, social support and NEI and PEI, country-specific univariate regression models were constructed to model NEI or PEI as a function of each SS variable and the food insecurity groups (see online supplementary material, Supplemental 
Table 1 Sociodemographic characteristics, food insecurity, types of social support and mental well-being in sub-Saharan Africa, 2014-2016

\begin{tabular}{|c|c|c|c|c|c|c|c|c|}
\hline & \multicolumn{2}{|c|}{2014} & \multicolumn{2}{|c|}{2015} & \multicolumn{2}{|c|}{2016} & \multicolumn{2}{|c|}{ All } \\
\hline & $\begin{array}{l}\% \text { or } \\
\text { mean }\end{array}$ & $\begin{array}{c}95 \% \mathrm{Cl} \text { or } \\
\text { SE }\end{array}$ & $\begin{array}{l}\% \text { or } \\
\text { mean }\end{array}$ & $\begin{array}{c}95 \% \mathrm{Cl} \text { or } \\
\text { SE }\end{array}$ & $\begin{array}{l}\% \text { or } \\
\text { mean }\end{array}$ & $\begin{array}{c}95 \% \mathrm{Cl} \text { or } \\
\mathrm{SE}\end{array}$ & $\begin{array}{l}\% \text { or } \\
\text { mean }\end{array}$ & $\begin{array}{c}95 \% \mathrm{Cl} \text { or } \\
\text { SE }\end{array}$ \\
\hline \multicolumn{9}{|l|}{ Characteristics of survey respondents } \\
\hline Age (years) & $34 \cdot 2$ & 0.11 & 34.0 & 0.13 & 34.3 & $0 \cdot 12$ & $34 \cdot 1$ & 0.07 \\
\hline Male $(\%)$ & $49 \cdot 1$ & $48 \cdot 5,49 \cdot 8$ & $48 \cdot 8$ & $48.2,49.5$ & 48.9 & $48 \cdot 2,49 \cdot 5$ & 48.9 & $48.5,49 \cdot 3$ \\
\hline Married $(\%)$ & 51.6 & $50 \cdot 9,52 \cdot 4$ & 52.9 & $52 \cdot 1,53 \cdot 8$ & 53.0 & $52 \cdot 3,53 \cdot 8$ & 52.5 & $52 \cdot 0,53 \cdot 0$ \\
\hline Elementary education or less (\%) & $66 \cdot 1$ & $65 \cdot 3,67 \cdot 0$ & $66 \cdot 7$ & $65 \cdot 8,67.5$ & $65 \cdot 2$ & $64 \cdot 4,65 \cdot 9$ & 66.0 & $65 \cdot 5,66.5$ \\
\hline No. of children under 15 years & 2.5 & 0.03 & $3 \cdot 1$ & 0.03 & 3.0 & 0.03 & $2 \cdot 8$ & 0.02 \\
\hline No. of people over 15 years & 3.9 & 0.02 & 3.9 & 0.02 & 3.6 & 0.02 & 3.8 & 0.01 \\
\hline Rural residency (\%) & 38.9 & $37.5,40 \cdot 4$ & 34.6 & $33 \cdot 3,35 \cdot 9$ & 34.4 & $33 \cdot 1,35 \cdot 7$ & $36 \cdot 1$ & $35 \cdot 3,36 \cdot 9$ \\
\hline \multicolumn{9}{|l|}{ FIES score range } \\
\hline 0 & $24 \cdot 0$ & $23 \cdot 2,24 \cdot 8$ & 19.7 & $18 \cdot 9,20 \cdot 6$ & $16 \cdot 2$ & $15 \cdot 5,16 \cdot 8$ & $20 \cdot 1$ & $19 \cdot 6,20.5$ \\
\hline $1-3$ & $19 \cdot 4$ & $18 \cdot 8,20 \cdot 1$ & $18 \cdot 3$ & $17 \cdot 6,19 \cdot 0$ & $15 \cdot 9$ & $15 \cdot 3,16 \cdot 5$ & $17 \cdot 9$ & $17 \cdot 5,18.3$ \\
\hline $4-6$ & $22 \cdot 9$ & $22 \cdot 3,23 \cdot 5$ & 24.7 & $23 \cdot 9,25 \cdot 5$ & $22 \cdot 7$ & $22 \cdot 1,23 \cdot 3$ & 23.4 & $23 \cdot 0,23.8$ \\
\hline $7-8$ & 33.7 & $32 \cdot 8,34 \cdot 5$ & $37 \cdot 3$ & $36 \cdot 2,38 \cdot 3$ & $45 \cdot 2$ & $44 \cdot 3,46 \cdot 1$ & $38 \cdot 6$ & $38 \cdot 0,39.2$ \\
\hline \multicolumn{9}{|l|}{ Social support } \\
\hline Perceived (\%) & 73.1 & $72 \cdot 3,73 \cdot 8$ & $69 \cdot 1$ & $68 \cdot 3,69 \cdot 9$ & $69 \cdot 1$ & $68.3,69.9$ & $70 \cdot 5$ & $70 \cdot 0,71 \cdot 0$ \\
\hline Foreign perceived (\%) & $36 \cdot 8$ & $36 \cdot 0,37 \cdot 7$ & 37.8 & $36 \cdot 9,38 \cdot 7$ & & N/A & $37 \cdot 3$ & $36 \cdot 7,37.9$ \\
\hline Received (\%) & $39 \cdot 0$ & $38 \cdot 0,39 \cdot 9$ & 35.6 & $34 \cdot 6,36 \cdot 7$ & $27 \cdot 8$ & $27 \cdot 1,28 \cdot 6$ & $34 \cdot 2$ & $33.7,34.8$ \\
\hline Given (\%) & 30.5 & $29 \cdot 6,31.4$ & 31.0 & $29 \cdot 9,32 \cdot 0$ & 28.9 & $28 \cdot 2,29 \cdot 7$ & 30.1 & $29 \cdot 6,30 \cdot 7$ \\
\hline Integrative (\%) & $70 \cdot 2$ & $69 \cdot 2,71 \cdot 2$ & 73.2 & $72 \cdot 2,74 \cdot 1$ & $73 \cdot 8$ & $73 \cdot 0,74 \cdot 7$ & $72 \cdot 4$ & $71 \cdot 8,72 \cdot 9$ \\
\hline Emotional (\%) & $62 \cdot 0$ & $61 \cdot 1,62 \cdot 8$ & 63.3 & $62 \cdot 4,64 \cdot 1$ & & $\mathrm{~N} / \mathrm{A}$ & 62.6 & $62 \cdot 0,63 \cdot 2$ \\
\hline \multicolumn{9}{|l|}{ Mental well-being } \\
\hline NEI & $30 \cdot 1$ & 0.26 & $30 \cdot 8$ & 0.31 & 35.5 & 0.28 & $32 \cdot 1$ & 0.18 \\
\hline PEI & $67 \cdot 8$ & 0.25 & $68 \cdot 1$ & 0.28 & & N/A & 68.0 & 0.19 \\
\hline
\end{tabular}

FIES, Food Insecurity Experience Scale; NEI, Negative Experience Index; PEI, Positive Experience Index; N/A, not applicable.

Figs 2-4). To account for the observed country-specific relationships, the random intercept and random coefficient models were applied at country level, because the former were significantly better than the latter in likelihood ratio tests in all the outcome-exposure paired models. To further explore potential interaction effects between food security status and social support (i.e. whether the presence of different types of social support modifies the association between food security status and mental wellbeing), a categorical-by-categorical interaction term was included in the random intercept and random coefficient models. Data for all years were pooled because the survey year did not modify the relationship between food insecurity, social support and mental well-being, and adding year as a third level to the model did not improve estimation significantly.

To adjust for potential confounding effects, a number of covariates were included in the multivariable models: respondents' age, sex, education, employment status, place of residence, number of children under 15 years in the household, number of adults in the household, country-specific household income quintiles and survey month.

\section{Results}

The total sample size, pooling all countries for the three years, was 102235 . Of the total sample, 2673 (2.6\%) were excluded from the analysis for answering 'don't know' or 'refused to answer' to at least one of the five questions that compose NEI. The excluded respondents for PEI analysis were 36987 (36.2\%), primarily because the question 'learning or doing something interesting' had not been asked in all countries in 2016. The number of respondents with a missing value to any FIES question was 3250 (3.2\%). Among the SS variables, the missing values ranged from $0.3 \%$ for perceived SS to $2 \cdot 8 \%$ for given SS. Foreign perceived and emotional SS were not asked in 2016 in all thirty-nine countries, resulting in 33708 (33.0\%) and 33468 (32.7\%) missing values for these two variables. Overall, the total numbers of respondents included in the NEI and PEI analyses were 96796 (94.7\%) and 63504 (62.1\%), respectively.

The distribution of sociodemographic characteristics, food insecurity, SS variables and mental well-being in subSaharan Africa is presented in Table 1. Almost 39\% of the respondents were in the most severe food insecurity group, based on their raw scores of $7-8$. About $70 \%$ of the respondents had perceived (70.5\%) and integrative SS $(72 \cdot 4 \%)$, while emotional SS was affirmed by $62.6 \%$ of the respondents. Fewer than $40 \%$ of the respondents affirmed foreign perceived SS $(37.3 \%)$, received SS (34.3\%) or given SS (30.1\%). The weighted mean NEI and PEI scores were $32 \cdot 1$ (SE 0.18) and 68.0 (SE 0.19), respectively.

Figures 1 and 2 present the adjusted marginal relationships between food insecurity, SS types and their interaction with NEI and PEI, respectively (see the online supplementary material, Supplemental Tables 3 and 4, for 
(a)

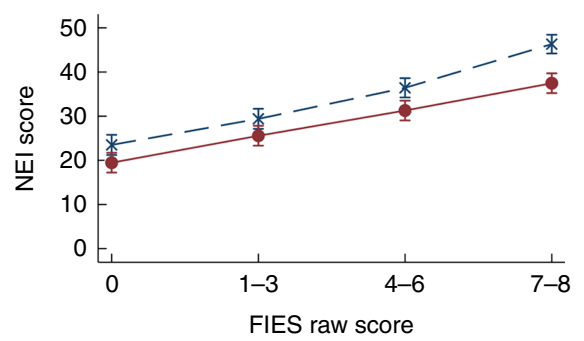

(c)

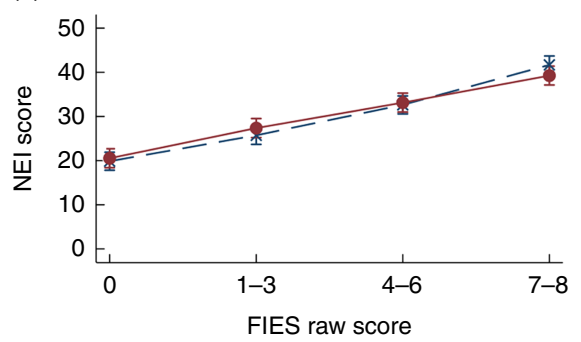

(e)

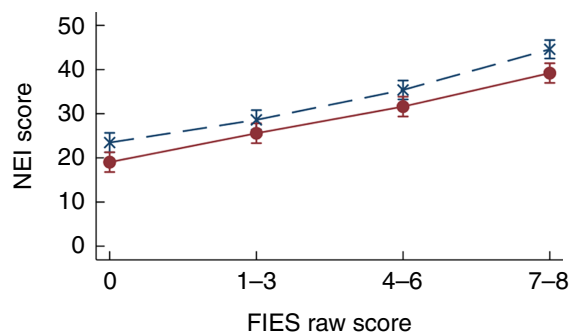

(b)

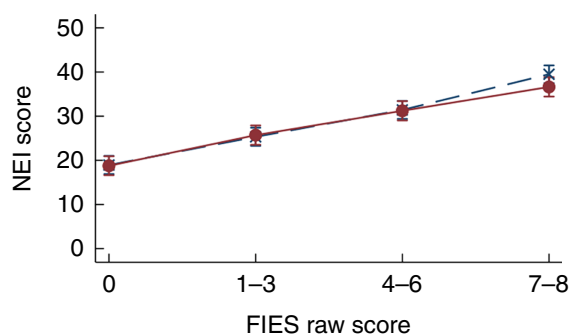

(d)

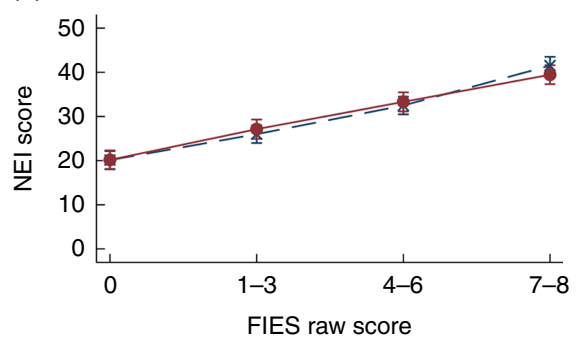

(f)

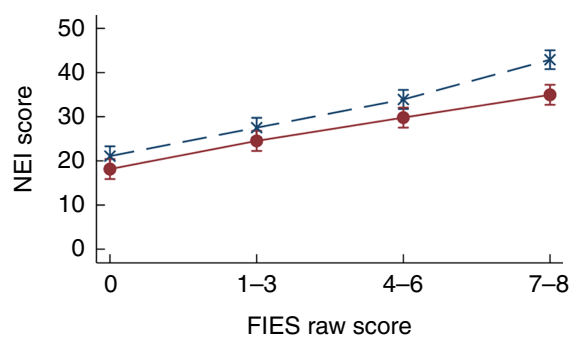

Fig. 1 The marginal effect of food insecurity and types of social support (SS; --x--, no support; -----, yes support) on mental wellbeing assessed by Negative Experience Index (NEI) score (mean values with their standard errors represented by vertical bars) in sub-Saharan Africa, 2014-2016: (a) perceived SS; (b) foreign perceived SS; (c) received SS; (d) given SS; (e) integrative SS; (f) emotional SS. The relationship is adjusted for respondent's age, sex, number of children under 15 years, number of adults over 15 years, education, employment status, place of residence, country-specific income quintiles and survey month. Food insecurity was measured using the Food Insecurity Experience Scale (FIES; raw scores represent four levels of food insecurity severity, with 7-8 being the most severe)

the detailed coefficients, $95 \% \mathrm{CI}$ and random effect of the unadjusted and adjusted relationships). There are three major findings depicted in Figs 1 and 2 that are important to highlight. First, there was a dose-response association between increasing severity of food insecurity and higher NEI and lower PEI. The second finding was that social support appeared to improve mental well-being across the FIES raw score groups, as demonstrated by the distance between those with (red solid line) and without social support (blue dashed line). The NEI scores among people who reported having perceived, integrative or emotional SS were significantly lower by 3-4 points. The PEI scores were higher by 6-9 points for people having perceived, integrative or emotional SS and by 3 points for those with foreign perceived SS. Neither NEI nor PEI differed significantly by received or given SS. The final point highlighted by Figs 1 and 2 was that the modifying effect of social support was significantly greater among those with the most severe food insecurity, except for integrative SS for NEI and perceived SS for PEI.

\section{Discussion and conclusion}

Using nationally representative large-scale survey data, we examined the relationship between food security status, multiple types of social support and mental well-being in thirty-nine sub-Saharan African countries. Echoing the findings of previous studies, our results showed that food security status independently predicted mental well-being in a dose-responsive manner ${ }^{(8)}$. In general, social support was associated with better reported mental well-being, with the relationship being strongest among adults with the most severe food insecurity. Specifically, the presence of perceived, integrative and emotional social support consistently predicted better mental well-being, i.e. higher PEI and lower NEI scores, while neither given nor received social support was a significant predictor of mental well-being. This finding is in line with a large body of research providing strong evidence of the link between availability of support and lower levels of both physical and psychological morbidity ${ }^{(10)}$. The lack of association 
(a)

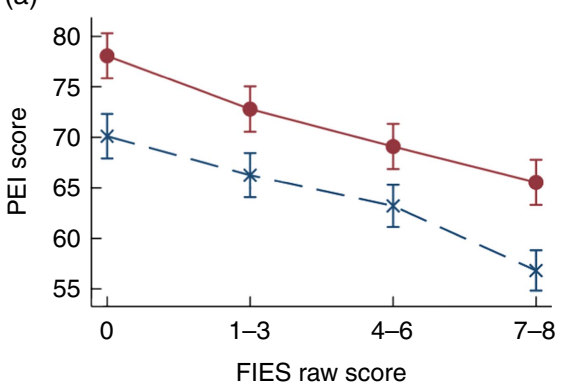

(c)

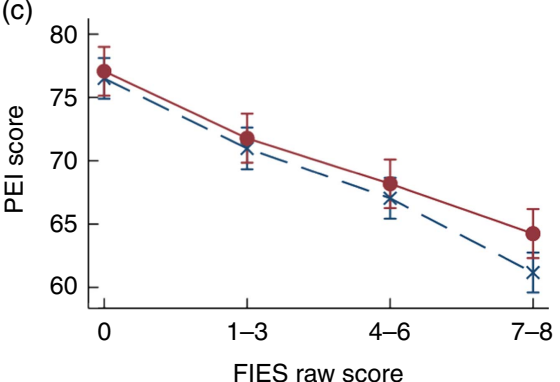

(e)

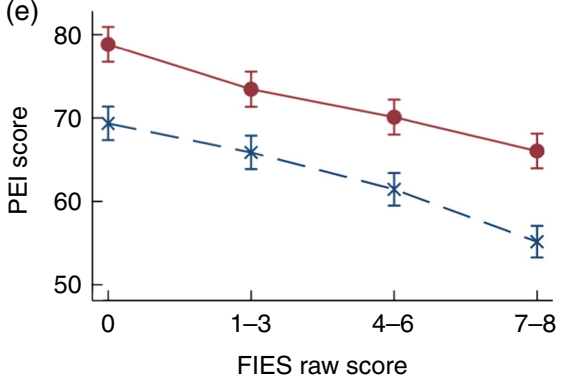

(b)

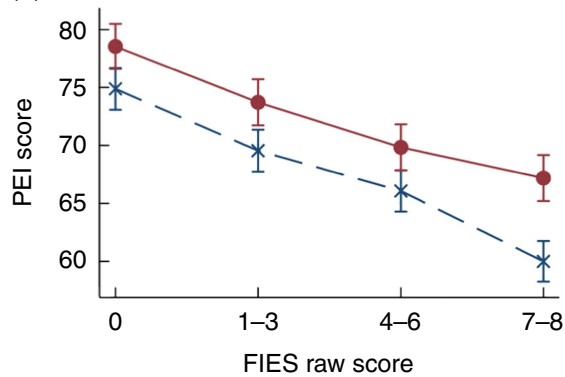

(d)

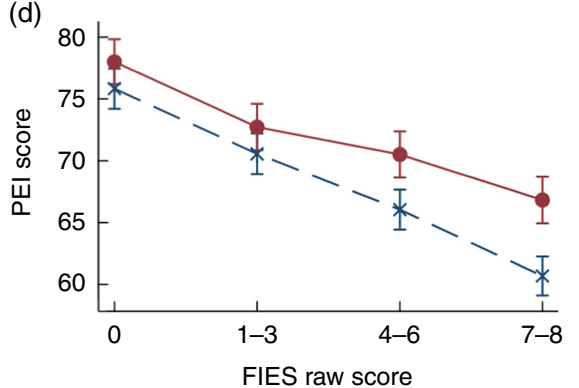

(f)

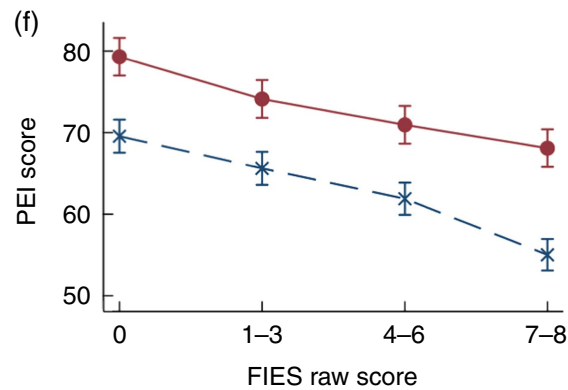

Fig. 2 The marginal effect of food insecurity and types of social support (SS; --x--, no support; -----, yes support) on mental wellbeing assessed by Positive Experience Index (PEI) score (mean values with their standard errors represented by vertical bars) in sub-Saharan Africa, 2014-2016: (a) perceived SS; (b) foreign perceived SS; (c) received SS; (d) given SS; (e) integrative SS; (f) emotional SS. The relationship is adjusted for respondent's age, sex, number of children under 15 years, number of adults over 15 years, education, employment status, place of residence, country-specific income quintiles and survey month. Food insecurity was measured using the Food Insecurity Experience Scale (FIES; raw scores represent four levels of food insecurity severity, with 7-8 being the most severe)

between mental well-being and received support is in line with the so-called 'paradox', well documented and tested in the social support literature, where receipt of support either has no significant relationship to health or has actual negative effects ${ }^{(24)}$. This may be explained by support being received only when one is worse off, or else the potential positive impact of the support is negated by feelings of shame, dependence or awareness of the gravity of one's situation ${ }^{(25,26)}$. The fact that the modifying effect of social support was found to be strongest among the most severely food insecure confirms previous studies supporting the stress-buffering model, whereby social support will have its greatest impact among people facing the most stressful conditions ${ }^{(10)}$.

The modifying role of social support revealed by the present study confirms similar results found in research conducted on specific groups of vulnerable sub-populations, including pregnant women in Uganda ${ }^{(15)}$, HIV- infected adults in Uganda ${ }^{(14)}$ and Latino minorities with type 2 diabetes in the USA ${ }^{(16)}$. Each of the studies used a measure based on a social support scale ${ }^{(27,28)}$, and in all three, social support protected individuals against negative impacts of food insecurity on depressive symptoms. The two studies conducted in Uganda additionally explored interactions between specific support types and food security status but reported different results: the moderating effect of instrumental, but not emotional social support, was stronger among HIV-infected women with moderate or severe food insecurity than it was for foodsecure women in Uganda ${ }^{(14)}$. However, such modification did not differ by type of social support among pregnant women in Uganda ${ }^{(15)}$. In another study from Canada, the prevalence of mental illness was higher among food-insecure individuals. This relationship was stronger among women and those who were socially isolated ${ }^{(29)}$. These inconsistencies suggest the need for continued 
research into the nature of social support's modifying effect among specific sub-populations, but do not detract from the clear evidence of its significant role in the relationship between food security status and mental wellbeing.

Social support cannot be assumed to act uniformly in relation to health. Its role may differ depending on the type of support, the specific health outcome considered, the level of congruity between the support needed and what is provided, and the social and cultural context. Given this complexity, it is essential to maintain distinctions among types of support, to consider potential interactions with relevant stress factors, and to avoid drawing generalizations about the nature of social support's relationship with health outside a limited context. While these important considerations have been taken into account previously by the small number of studies referenced above, our study has been able to expand those findings beyond a small sub-population and single mental health outcomes. We used standardized measures of food security status, social support and mental well-being, which were applied across thirty-nine countries and three years of data collection, allowing for data to be pooled and used to produce regional results for sub-Saharan Africa. Moreover, rigorous multilevel models were applied to account for country-level differences in the prevalence of food insecurity, the associations between food insecurity, social support and mental well-being, and possible unmeasured country-level confounders.

There are several limitations of the analysis to be noted. Data from GWP surveys are cross-sectional by nature, meaning that causality cannot be determined. Different reference periods were used for the measures of mental well-being (yesterday), food insecurity (past 12 months) and social support (past 12 months); the observed association may therefore be attenuated due to day-to-day variability of experiences associated with mental wellbeing. Self-reported data may also result in reporting biases, the direction of which is unknown. The high exclusion rate in surveys of certain countries and years due to security reasons, as well as missing data for some SS and PEI variables, may compromise the representativeness of our study findings (see online supplementary material, Supplemental Table 5). However, a sensitivity analysis suggested the results were robust if using PEI derived from the at least four available questions by GWP (Supplemental Table 6). Heterogeneity observed in country-specific associations, especially with regard to PEI (Supplemental Fig. 3), may count against the overall marginal effect when combining all the countries, even when country-level effects are adjusted. However, the concern may be minimal, according to the almost identical results of our sensitivity analysis that excluded the outlier countries (Supplemental Fig. 5). Finally, the lack of information regarding community-level social support prevented us from examining the potential mitigation effect of social support on adverse mental health outcomes at broader ecological levels.

In sub-Saharan Africa, addressing the extremely high prevalence of severe food insecurity must be made a priority of national governments. Social support's role in protecting people against the stress of being food insecure may have positive implications for their mental well-being, especially in a context where health-care resources are failing to meet needs. The interaction shown in the present study between food security and several types of social support, which was significant only among the most severely food insecure, suggests a heavy dependence on informal support systems, as opposed to reliance on government social protection programmes, to cope in the face of hardship. Governments cannot rely on social support to offset the stress caused by food insecurity and should devote increased effort to strengthening social protection systems to improve access to adequate food. Lack of social support should, however, be recognized as an aspect of vulnerability and included among other indicators in routine assessment and monitoring. Given the limited evidence supporting specific social support interventions $^{(28)}$, future research is needed to better understand its important role in relation to mental well-being, particularly among food-insecure populations.

\section{Acknowledgements}

Acknowledgements: The authors acknowledge FAO's Voices of the Hungry Project for having granted access to the full GWP data set and supporting materials, which allowed the present study to be conducted. They appreciate the help from Kristen Cox for her work on presenting descriptive measures of the data. Y.W.H.'s time is supported by the National Institute on Drug Abuse (grant numbers P50: P50 DA010075, P50: P50 DA039838 and T32 DA017629). The content is solely the responsibility of the authors and does not necessarily represent the official views of the National Institute on Drug Abuse or the National Institutes of Health. Financial support: This research received no specific grant from any funding agency in the public, commercial or not-for-profit sectors. Conflict of interest: None. Authorship: M.N. conceptualized the research question. M.N. requested data through a competitive call for proposals by the FAO's Voices of the Hungry Project and conducted data analysis. D.C.M. managed the database and conducted exploratory data analysis. M.N., M.M. and T.B. conducted the literature review and prepared the first draft of the manuscript. D.C.M., Y.W.H. and H.M.-Q. provided technical support on study methods, insights on results interpretation and revisions to the manuscript. All authors read and approved the final manuscript. Ethics of buman subject participation: The Gallup World Poll data granted by FAO does not include any identifiable information. Therefore, the study 
is not considered human subjects research defined by the Department of Health and Human Services.

\section{Supplementary material}

To view supplementary material for this article, please visit https://doi.org/10.1017/S136898001800277X

\section{References}

1. World Health Organization (2018) Mental health: Strengthening our response. Fact sheet. http://www.who.int/news$\mathrm{room} /$ fact-sheets/detail/mental-health-strengthening-ourresponse (accessed October 2018).

2. World Health Organization (2017) Depression and Other Common Mental Disorders: Global Health Estimates. Geneva: WHO

3. Saxena S, Thornicroft G, Knapp M et al. (2007) Resources for mental health: scarcity, inequity, and inefficiency. Lancet 370, 878-889.

4. Wilkinson RG \& Marmot M (2003) Social Determinants of Health: The Solid Facts. Copenhagen: WHO Regional Office for Europe.

5. Roncarolo F \& Potvin L (2016) Food insecurity as a symptom of a social disease: analyzing a social problem from a medical perspective. Can Fam Physician 62, 291-292.

6. Vozoris NT \& Tarasuk VS (2003) Household food insufficiency is associated with poorer health. J Nutr 133, 120-126.

7. Lund C, Breen A, Flisher AJ et al. (2010) Poverty and common mental disorders in low and middle income countries: a systematic review. Soc Sci Med 71, 517-528.

8. Jones AD (2017) Food insecurity and mental health status: a global analysis of 149 countries. Am J Prev Med 53, 264-273.

9. Weaver LJ \& Hadley C (2009) Moving beyond hunger and nutrition: a systematic review of the evidence linking food insecurity and mental health in developing countries. Ecol Food Nutr 48, 263-284.

10. Gottlieb BH, Cohen S, Underwood LG et al. (2000) Social Support Measurement and Intervention: A Guide for Health and Social Scientists. New York: Oxford University Press, Inc.

11. Uchino BN (2009) Understanding the links between social support and physical health: a life-span perspective with emphasis on the separability of perceived and received support. Perspect Psychol Sci 4, 236-255.

12. Ashe KM \& Lapane KL (2017) Food insecurity and obesity: exploring the role of social support. $J$ Womens Health (Larchmt) 27, 651-658.

13. dos Santos IN, de Jesus Damião J, da Fonseca MdJM et al. (2018) Food insecurity and social support in families of children with sickle-cell disease. J Pediatr (Rio J). Published online: 15 March 2018. doi: 10.1016/j.jped.2018.01.005.

14. Tsai AC, Bangsberg DR, Frongillo EA et al. (2012) Food insecurity, depression and the modifying role of social support among people living with HIV/AIDS in rural Uganda. Soc Sci Med 74, 2012-2019.
15. Natamba BK, Mehta S, Achan J et al. (2017) The association between food insecurity and depressive symptoms severity among pregnant women differs by social support category: a cross-sectional study. Matern Child Nutr 13, e12351.

16. Kollannoor-Samuel G, Wagner J, Damio G et al. (2011) Social support modifies the association between household food insecurity and depression among Latinos with uncontrolled type 2 diabetes. J Immigr Minor Health $\mathbf{1 3}$, 982.

17. Food and Agriculture Organization of the United Nations, International Fund for Agricultural Development, UNICEF et al. (2017) The State of Food Security and Nutrition in the World 2017: Building Resilience for Peace and Food Security. Rome: FAO.

18. Nanama S \& Frongillo EA (2012) Altered social cohesion and adverse psychological experiences with chronic food insecurity in the non-market economy and complex households of Burkina Faso. Soc Sci Med 74, 444-451.

19. European Report on Development (2010) ERD 2010 Background paper: Formal and informal social protection in SubSaharan Africa. https://ec.europa.eu/europeaid/erd-2010background-paper-formal-and-informal-social-protectionsub-saharan-africa-abena-d-oduro-0_en (accessed October 2018).

20. Gallup Inc. (2017) Worldwide Research Methodology and Codebook. https://data-services.hosting.nyu.edu/wp-con tent/uploads/2017/10/World_Poll_Methodology_102717. pdf (accessed October 2018).

21. Gallup Inc. (2018) Country Data Set Details Gallup Worldwide Research Data Collected 2005/2006-2018. https:// www.gallup.com/file/services/177797/World_Poll_Dataset_ Details_022318.pdf (accessed October 2018).

22. Cafiero C, Viviani S \& Nord M (2018) Food security measurement in a global context: the Food Insecurity Experience Scale. Measurement 116, 146-152.

23. Wolter K (2007) Introduction to Variance Estimation. New York: Springer.

24. Nurullah AS (2012) Received and provided social support: a review of current evidence and future directions. Am J Health Stud 27, 173-188.

25. Seidman G, Shrout PE \& Bolger N (2006) Why is enacted social support associated with increased distress? Using simulation to test two possible sources of spuriousness. Pers Soc Psychol Bull 32, 52-65.

26. Lepore SJ, Glaser DB \& Roberts KJ (2008) On the positive relation between received social support and negative affect: a test of the triage and self-esteem threat models in women with breast cancer. Psychooncology 17, 1210-1215.

27. Zimet GD, Dahlem NW, Zimet SG et al. (1988) The multidimensional scale of perceived social support. J Pers Assess 52, 30-41.

28. Broadhead W, Gehlbach SH, De Gruy FV et al. (1988) The Duke-UNC Functional Social Support Questionnaire: measurement of social support in family medicine patients. Med Care 26, 709-723.

29. Martin M, Maddocks E, Chen Y et al. (2016) Food insecurity and mental illness: disproportionate impacts in the context of perceived stress and social isolation. Public Health 132, 86-91. 